\title{
Immediate extubation after esophagectomy with three-field lymphadenectomy enables early ambulation in patients with thoracic esophageal cancer
}

\author{
Takeharu Imai $^{1,2} \cdot$ Tetsuya Abe $^{1}$ (D) Norihisa Uemura ${ }^{1} \cdot$ Kazuhiro Yoshida $^{2} \cdot$ Yasuhiro Shimizu $^{1}$
}

Received: 4 September 2017 / Accepted: 6 March 2018 / Published online: 12 March 2018

(c) The Japan Esophageal Society and Springer Japan KK, part of Springer Nature 2018

\begin{abstract}
Background We retrospectively compared the effects of immediate extubation (IE) in the operating room with those of overnight mechanical ventilation (MV) after radical transthoracic esophagectomy with 3-field lymphadenectomy in patients with thoracic esophageal cancer.

Methods A total of 96 patients were evaluated. 48 patients were extubated in the operating room after surgery (IE group). The other 48 patients were extubated on the following morning (MV group). The propensity score-matching method was used to assemble a well-balanced cohort. Clinical and postoperative outcomes were investigated in each group. We also compared postoperative laboratory parameters between groups.

Results The rate of ambulation on postoperative day (POD) 1 was significantly higher in the IE group compared with that in the MV group (50 vs $19 \%$, respectively, $p=0.003$ ). Moreover, the rate of catecholamine use in the ICU was significantly lower in the IE group compared with that in the MV group (15 vs 65\%, respectively, $p<0.001$ ). With regard to postoperative respiratory management, there were no significant differences between groups. The length of ICU stay after esophagectomy was significantly shorter in the IE group compared with that in the MV group $(p=0.01)$, whereas the length of postoperative hospital stay was similar between groups $(p=0.265)$. There were also no significant differences in the incidence of postoperative complications.

Conclusions IE in the operating room is not only safe and feasible, even after transthoracic esophagectomy with radical 3 -field lymphadenectomy, but also contributes to decrease in catecholamine use, to increase in ambulation on POD 1 and to shorten the ICU stay.
\end{abstract}

Keywords Immediate extubation · Esophagectomy · Esophageal cancer

\section{Introduction}

Despite recent advances in perioperative management, esophagectomy for esophageal cancer is a highly invasive surgical procedure that is associated with high morbidity and mortality [1-3]. Pulmonary complications are the most common morbidity after radical esophagectomy $[4$,

Tetsuya Abe

tabe@aichi-cc.jp

1 The Department of Gastroenterological Surgery, Aichi Cancer Center Hospital, 1-1 Kanokoden, Chikusa-ku, Nagoya, Aichi 464-8681, Japan

2 The Department of Surgical Oncology, Gifu University, Graduate School of Medicine, Gifu 501-1194, Japan
5]. The incidence of major respiratory complications can reach $30 \%$ or higher, even in high-volume centers $[6,7]$. Because respiratory failure due to pulmonary complications is a major cause of postoperative mortality, efforts have been made to reduce pulmonary complications in the perioperative management of esophagectomy, including preoperative anti-smoking education, perioperative aggressive nutrition support, inspiratory muscle training, postoperative epidural analgesia, minimally invasive surgery, and perioperative methylprednisolone and neutrophil elastase inhibitor administration [7-10].

In early perioperative respiratory management after radical esophagectomy, the use of overnight mechanical ventilation (MV) or immediate extubation (IE) in the operating room varies based on institutional preference. Prophylactic overnight MV was historically introduced to 
prevent aspiration, secure the airway, and allow for adequate pain control during the early perioperative period $[11,12]$. However, overnight MV is also likely to prompt pulmonary complications, such as ventilator-associated pneumonia, barotrauma, and endotracheal tube-related problems [13]. Moreover, overnight MV is routinely carried out with the use of sedatives, which have the potential to impede early ambulation and early recovery after esophagectomy. Although several studies have demonstrated that IE in the operating room is feasible, safe, and associated with lower morbidity for patients with thoracic esophageal cancer after transthoracic esophagectomy with radical lymphadenectomy [14-18], the timing of extubation after esophagectomy remains controversial, with no common practice, even among experienced centers. We hypothesized that IE would also facilitate earlier recovery after esophagectomy compared with overnight MV in patients with thoracic esophageal cancer after transthoracic esophagectomy with radical 3-field lymphadenectomy. To test this hypothesis, we compared the postoperative complication rates and short-term outcomes after radical transthoracic esophagectomy with 3-field lymphadenectomy in patients with thoracic esophageal cancer managed with overnight MV versus IE.

\section{Materials and methods}

\section{Study design}

The design of this study is a retrospective cohort study using a historical control. Between January 2010 and April 2013, 173 patients with thoracic esophageal cancer underwent transthoracic esophagectomy at the Aichi Cancer Center Hospital. Of these, 31 patients were excluded because they underwent radical esophagectomy with 2-field lymph node dissection. Of the remaining 142 patients, 13 who underwent thoracoscopic esophagectomy and laparoscopic surgery and 9 who underwent MV later than postoperative day 1 were excluded. Finally, we analyzed the records of the 120 patients who underwent esophagectomy via thoracotomy and laparotomy with extended radical 3-field lymph node dissection. Of these, 60 patients up to July 2011 were managed with overnight MV in the surgical intensive care unit (ICU) after esophagectomy (MV group). However, with the aim of changing respiratory management, the policy of early postoperative respiratory management was changed from overnight MV to IE in the operating room in August 2011. Therefore, the remaining 60 patients were extubated immediately after esophagectomy in the operating room (IE group). Clinical data were collected from a prospectively maintained database of patients at the Aichi Cancer Center Hospital. This study was approved by the review board of the Aichi Cancer Center Hospital.
Surgical procedure of transthoracic esophagectomy

All patients with thoracic esophageal cancer underwent radical subtotal esophagectomy with 3-field lymphadenectomy via right thoracotomy and laparotomy. We routinely performed cervical and upper abdominal lymph node dissection before thoracotomy, unless the preoperative assessment suggested the presence of unresectable lesions in the mediastinum. We routinely performed the dissection around the recurrent laryngeal nerves from the cervix to the upper mediastinum, as caudal as possible. Then further dissection around the recurrent laryngeal nerves was performed to maintain continuity with the cervical dissection during thoracotomy. To avoid pulmonary complications, we preserved the bronchial arteries and the pulmonary branches of the vagus nerves during the procedures when possible. The thoracic duct was not routinely resected if the main tumor was not involved. Bowel continuity was routinely restored by a gastric tube via the retrosternal route, and subsequently, we created a feeding catheter gastrostomy with the round ligament of the liver for postoperative enteral nutrition [19].

\section{Perioperative management of patients with thoracic esophageal cancer}

Perioperative management of patients with thoracic esophageal cancer in the MV group was similar to that in the IE group, with the exception of the timing of extubation. For all patients, the following agents were administered orally or trans-luminally for 5 days before surgery: IMPACT ${ }^{\circledR}$ ( $1 \mathrm{kcal} / \mathrm{ml}$; AJINOMOTO Pharma, Tokyo, Japan) $750 \mathrm{ml} /$ day as an immune-enhanced diet and GFO ${ }^{\circledR}$ (Otsuka Pharmaceutical Factory, Inc., Tokushima, Japan) 3 packs/day $(1$ pack $=36 \mathrm{kcal}$; protein $3.6 \mathrm{~g}$, fat $0 \mathrm{~g}$, carbohydrates $6.0 \mathrm{~g}$, alimentary fiber $5.0 \mathrm{~g}, \mathrm{Na} 0.5 \mathrm{mg}$, galactosyl-sucrose $1.45 \mathrm{~g}$, glutamine $3.0 \mathrm{~g}$ ) as prebiotic therapy. All patients were persuaded to quit smoking 4-6 weeks preoperatively and underwent preoperative inspiratory muscle training with incentive spirometry. Patients without signs of esophageal stenosis received a regular diet, whereas those with signs of stenosis received a liquid diet orally or through a transnasal feeding catheter. All patients received oral care for 5 days before surgery. Thirty minutes before surgery, all patients received prophylactic antibiotics and methylprednisolone $(250 \mathrm{mg})$ as a single intravenous drip infusion. Differential lung ventilation was used during thoracotomy. For intraoperative fluid and circulation management, all patients received fluid replacement and catecholamines to keep the mean arterial pressure $>60 \mathrm{mmHg}$. After surgery, the patients in the MV group were transferred to the ICU 
and patients received a continuous intravenous infusion of propofol for sedation. All of the patients were extubated on the following morning, if feasible. The patients in the IE group were extubated in the operating room after surgery if the anesthesiologist approved. No special criteria for extubation existed, although excessive bleeding, lengthy operative time, hypothermia, hypercarbia, acidosis, hypothermia, and hemodynamic instability were considered relative contraindications to extubation. The patients were transferred to the ICU after the assessment of vocal cord palsy by bronchofiberscopy. Adequate postoperative analgesia was continued with dual epidural infusions using a patient-controlled analgesia system for 7 days, depending on the patient's wishes. The nasogastric tube was removed immediately after extubation in both groups. Enteral feeding was initiated $4 \mathrm{~h}$ post-surgery with IMPACT ${ }^{\circledR}(1 \mathrm{kcal} /$ $\mathrm{ml}$; AJINOMOTO Pharma, Tokyo, Japan) $250 \mathrm{ml} /$ day and was increased gradually to $1500 \mathrm{ml} /$ day by day 4 . On postoperative day 5 , Racol $\mathrm{RF}^{\circledR}(1 \mathrm{kcal} / \mathrm{ml}$; Otsuka Pharmaceutical Factory, Inc., Tokushima, Japan) 2000 ml/day was given. The chest drain was removed when the volume of pleural effusion became less than $200 \mathrm{ml} /$ day. Patients usually underwent an upper gastrointestinal series on postoperative day 8. Unless there was anastomotic leakage, oral intake was initiated on day 8 , with enteral feeding decreasing gradually as oral intake increased.

\section{Recording of clinical data}

Patient demographic data and clinical outcomes were compared between the MV and IE groups. Peripheral white blood cell and lymphocyte counts, C-reactive protein (CRP) levels, other biochemical parameters, catecholamine use in the ICU, ambulation on postoperative day 1, the need for bronchial toilet by fiber bronchoscopy, the incidence of reintubation and tracheotomy, and lengths of ICU and hospital stay were examined in each group. Clinical staging of tumors was classified according to the Tumor-Node-Metastasis (TNM) Classification of Malignant Tumors, seventh edition [20].

\section{Propensity score matching}

To control for potential differences in patient characteristics between groups, we used propensity score matching to assemble comparable groups. After estimating the propensity score of patients in the MV group, we matched each patient sequentially to a patient in the IE group who had the closest propensity score using simple 1:1 nearest neighbor matching. After propensity score calculation using logistic regression, patients were matched 1:1 using nearest neighbor matching with a caliper distance of 0.20 of the standard deviation of the logit of the propensity score. We included age, gender, body mass index, American Society of Anesthesiologists physical status (ASA-PS), depth of tumor invasion, and lymph node metastasis status as covariates. Eventually, 48 paired cases were matched from the cohort, and the 2 groups were comparable in patient characteristics (Fig. 1).

\section{Statistical analysis}

All statistical computations including propensity score matching were carried out with JMP 12 software (SAS
Fig. 1 Patient flowchart. Flowchart of the 48 paired cases who were matched from the cohort. 48 patients were managed with overnight MV in the surgical ICU (MV group) and other 48 patients were extubated immediately in the operating room (IE group) after esophagectomy

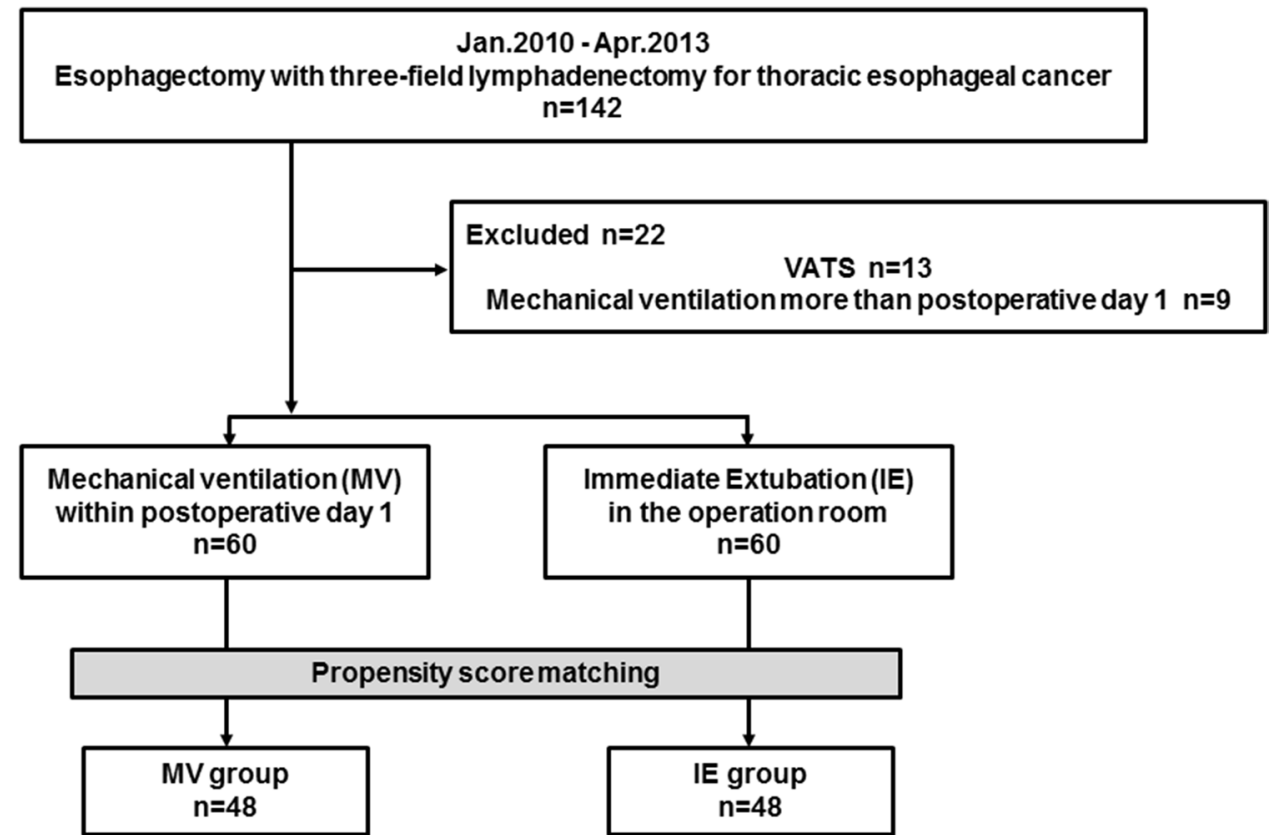


Institute Inc, Cary, NC, USA). The results were expressed as the median (interquartile range) for quantitative variables and as a percentage for qualitative variables. The $\chi^{2}$ test or the Fisher exact test was used for categorical variables. The nonparametric Wilcoxon rank sum test was used for continuous variables. A $p$ value of less than 0.05 was considered to be statistically significant.

\section{Results}

\section{Demographic and clinical characteristics}

Table 1 summarizes the demographic and clinical characteristics of the patients in the 2 groups. Both groups were well balanced for the variables of age, gender, body mass index, comorbidities, ASA-PS and clinical TNM stage, which were included in the propensity score derivation model (Table 1).

\section{Surgical characteristics}

Table 2 summarizes the surgical outcomes and postoperative course for each group. Several variables, including operative time, blood loss, intraoperative urine volume and intraoperative fluid volume were similar between groups. However, the intraoperative in-out balance was significantly lower in the IE group than in the MV group $(p=0.022)$.

\section{Early postoperative clinical outcomes}

Table 3 summarizes the early postoperative clinical outcomes of the patients in each group. The rate of ambulation on postoperative day 1 was significantly higher in the IE group than the MV group (50 vs 19\%, respectively; $p=0.003$ ). Moreover, the rate of catecholamine use in the ICU was significantly lower in the IE group than the MV group ( 15 vs $65 \%$, respectively; $p<0.001$ ). These differences may be attributed to the lack of sedative drug use. The rate of re-intubation and the need to perform bronchial toilet by fiber bronchoscopy in postoperative respiratory management were similar between groups. In the MV group, 5 patients
Table 1 Patient baseline characteristics

\begin{tabular}{|c|c|c|c|}
\hline Variable & MV group $(n=48)$ & IE group $(n=48)$ & $p$ \\
\hline Age [IQR] & $63[60-68.75]$ & $64.5[60-79.75]$ & 0.432 \\
\hline \multicolumn{4}{|l|}{ Gender } \\
\hline Male/female & $41 / 7$ & $42 / 6$ & 1.000 \\
\hline Body mass index $[\mathrm{IQR}]\left(\mathrm{kg} / \mathrm{m}^{2}\right)$ & $22.0[19.43-24.3]$ & $21.8[19.5-24.03]$ & 0.910 \\
\hline \multicolumn{4}{|l|}{ Smoking habits } \\
\hline Positive/negative & $40 / 8$ & $42 / 6$ & 0.772 \\
\hline \multicolumn{4}{|l|}{ Comorbidities } \\
\hline \multicolumn{4}{|l|}{ Diabetes mellitus } \\
\hline Positive/negative & $5 / 43$ & $1 / 47$ & 0.204 \\
\hline \multicolumn{4}{|l|}{ Cardiovascular disease } \\
\hline Positive/negative & $17 / 31$ & $20 / 28$ & 0.529 \\
\hline \multicolumn{4}{|l|}{ COPD } \\
\hline Positive/negative & $2 / 46$ & $1 / 47$ & 1.000 \\
\hline \multicolumn{4}{|l|}{ Nutrition status } \\
\hline \multicolumn{4}{|l|}{ Serum albumin (g/dl) } \\
\hline$<3.5 / \geq 3.5$ & $4 / 44$ & $7 / 41$ & 0.524 \\
\hline \multicolumn{4}{|l|}{ ASA-PS } \\
\hline $\mathrm{I} / \mathrm{II}$ & $27 / 21$ & $25 / 23$ & 0.682 \\
\hline \multicolumn{4}{|l|}{ cStage } \\
\hline I/II/III/IV & $3 / 14 / 28 / 3$ & $1 / 16 / 28 / 3$ & 0.907 \\
\hline \multicolumn{4}{|l|}{ Preoperative therapy } \\
\hline None & 1 & 1 & 0.723 \\
\hline $\begin{array}{l}\text { Neoadjuvant chemotherapy (FP/DCF/ } \\
\text { XELOX) }\end{array}$ & $37 / 9 / 1$ & $32 / 14 / 0$ & \\
\hline Definitive chemoradiation & 0 & 1 & \\
\hline
\end{tabular}

$I Q R$ interquartile range, ASA-PS American Society of Anesthesiologists physical status classification, $C O P D$ chronic obstructive pulmonary disease, $M V$ overnight mechanical ventilation, $I E$ immediate extubation 
Table 2 Surgical characteristics

\begin{tabular}{llll}
\hline Variable & MV group $(n=48)$ & IE group $(n=48)$ & $p$ \\
\hline Operative time (min), median [IQR] & $397.5[358.5-428.8]$ & $405[360-451.5]$ & 0.505 \\
Blood loss (ml), median [IQR] & $325[231.3-417.5]$ & $285[202.5-468.8]$ & 0.433 \\
Intraoperative urine volume (ml/kg/h), median [IQR] & $1.346[0.917-2.03]$ & $1.487[0.997-2.288]$ & $11.692[10.147-14.038]$ \\
Intraoperative fluid volume (ml/kg/h), median [IQR] & $13.069[11.347-15.01]$ & 0.174 \\
Intraoperative in-out balance (ml/kg/h), median [IQR] & $10.587[9.144-12.389]$ & $9.443[7.919-11.149]$ & 0.095 \\
\hline
\end{tabular}

$M V$ overnight mechanical ventilation, $I E$ immediate extubation, $I Q R$ interquartile range

Table 3 Clinical outcomes

\begin{tabular}{lllr}
\hline Variable & MV group $(n=48)$ & IE group $(n=48)$ & $p$ \\
\hline Catecholamine use in the ICU & $31(65 \%)$ & $7(15 \%)$ & $<0.001$ \\
Ambulation on postoperative day 1 & $9(19 \%)$ & $24(50 \%)$ & 0.003 \\
Suction of sputa by fiber bronchoscopy & $11(23 \%)$ & $11(23 \%)$ & 1.000 \\
Re-intubation & $5(10 \%)$ & $2(4 \%)$ & 0.435 \\
Tracheotomy & $5(10 \%)$ & $2(4 \%)$ & 0.435 \\
ICU stay after esophagectomy (days), median [IQR] & $3[3-4]$ & $3[2.75-3]$ & 0.01 \\
Postoperative hospital stay after esophagectomy & $23[18-40.75]$ & $22.5[16.5-27.75]$ & 0.265 \\
$\quad$ (days), median [IQR] & & & \\
\hline
\end{tabular}

With overlaps

$I C U$ intensive-care unit, $M V$ overnight mechanical ventilation, $I E$ immediate extubation, $I Q R$ interquartile range

underwent tracheotomy: 3 patients suffered from respiratory insufficiency with pneumonia, 1 patient suffered from respiratory insufficiency with bronchomediastinal fistula due to anastomotic leakage and 1 patient suffered from recurrent nerve palsy. In the IE group, 2 patients underwent tracheotomy: 1 patient suffered from respiratory insufficiency with pneumonia and 1 patient suffered from ventilator insufficiency with right phrenic nerve palsy. All patients who underwent tracheotomy required a re-intubation procedure. However, the incidence of tracheotomy was statistically similar between groups $(p=0.435)$. With regard to postoperative respiratory management, there were no significant differences between groups. The length of ICU stay after esophagectomy was significantly shorter in the IE group than the MV group ( $p=0.01$ ), whereas the length of postoperative hospital stay was similar between groups $(p=0.265)$.

\section{Postoperative laboratory parameters}

Figure 2 shows the postoperative laboratory parameters of the 2 groups. Although changes in the peripheral white blood cell counts, hemoglobin levels, platelet counts and lymphocyte counts were not significantly different between groups, the CRP level was significantly lower in the IE group than the MV group on postoperative day 1 . The serum albumin level and total bilirubin level were higher in the IE group on POD1. L-Asparate aminotransferase and L-alanine aminotransferase level were lower in the IE group on POD1. Creatinine level was higher in the IE group.

\section{Postoperative complications}

Postoperative complications are summarized in Table 4 . Pulmonary complications were defined by a clinical respiratory symptom, imaging findings, and the rise in inflammatory reaction with or without a pathogen. The total composite postoperative complication rate was similar between groups, 31 (65\%) in the MV group and 27 (56\%) in the IE group $(p=0.404)$. There were also no significant differences in the incidence of individual complications. Recurrent nerve palsy classified as Grade III or more was observed in 5 patients in the MV group (10\%) compared to 0 patients in the IE group $(0 \%)$, whilst the incidence of Grade I or II recurrent nerve palsy was comparable between groups $(8 \%)$. However, we never changed the extent of lymphadenectomy around the recurrent nerves in either group. Although intraoperative mortality was not observed in either group, in-hospital mortality was observed in 1 patient in the MV group (2\%) because of arterial bleeding from a tracheoinnominate artery fistula following tracheotomy on postoperative day 58 . 
Fig. 2 Postoperative laboratory parameters. Peripheral white blood cell counts (a), hemoglobin levels (b), platelet counts (c) and lymphocyte counts (d) were not significantly different between groups. The $\mathrm{C}$-reactive protein level (e) was significantly lower in the IE group on POD1. The serum albumin level (f) and total bilirubin level (g) were higher in the IE group on POD1. L-Asparate aminotransferase level (h) and L-alanine aminotransferase level (i) were lower in the IE group on POD1. Creatinine level (j) was higher in the IE group. Results are shown as mean $\pm 95 \%$ confidence intervals. Pre preoperative values. $P O D$ postoperative days. ${ }^{*} p<0.05$ a

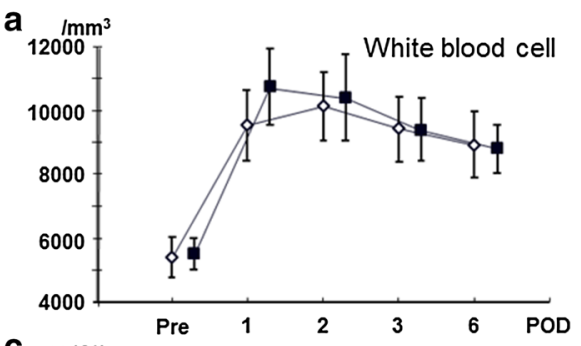

C $\times$

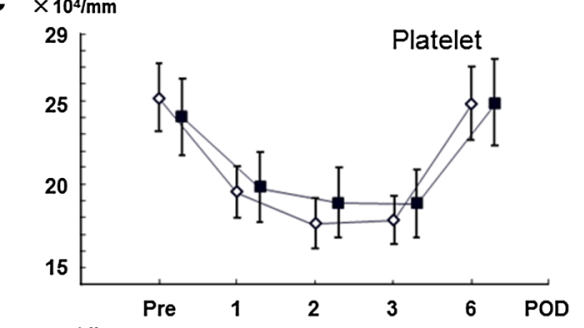

e

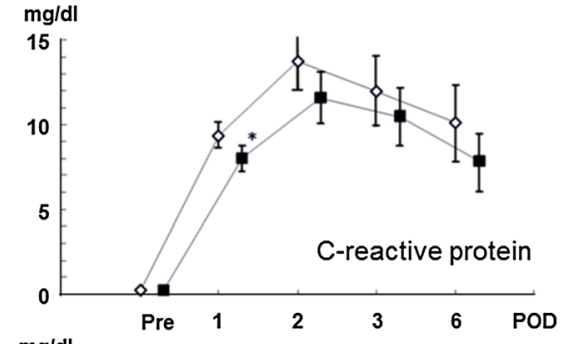

g

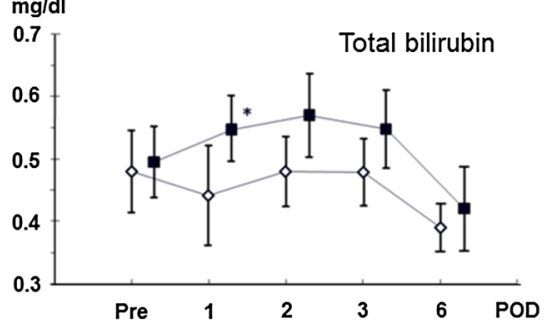

i

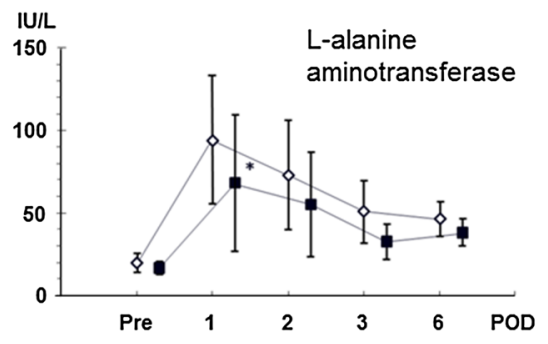

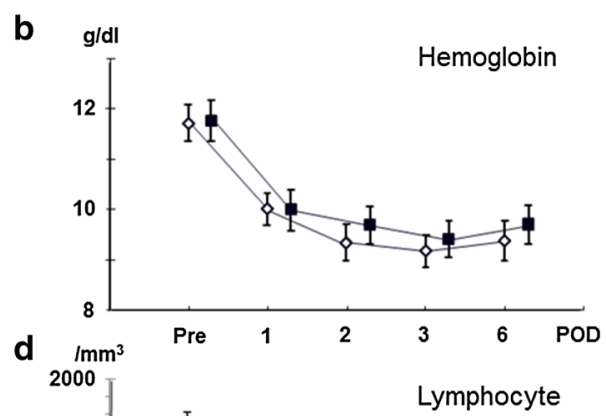

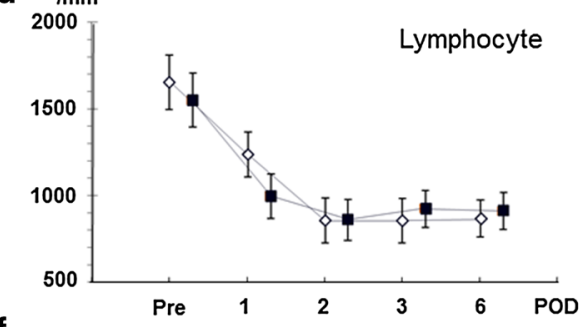

$f$

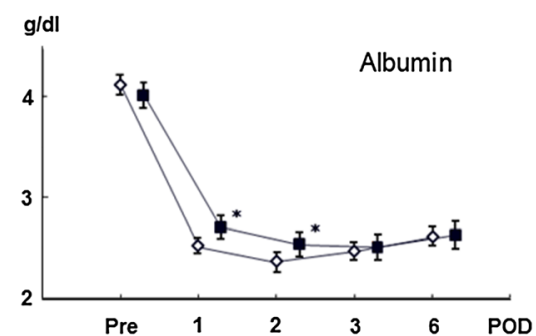

h
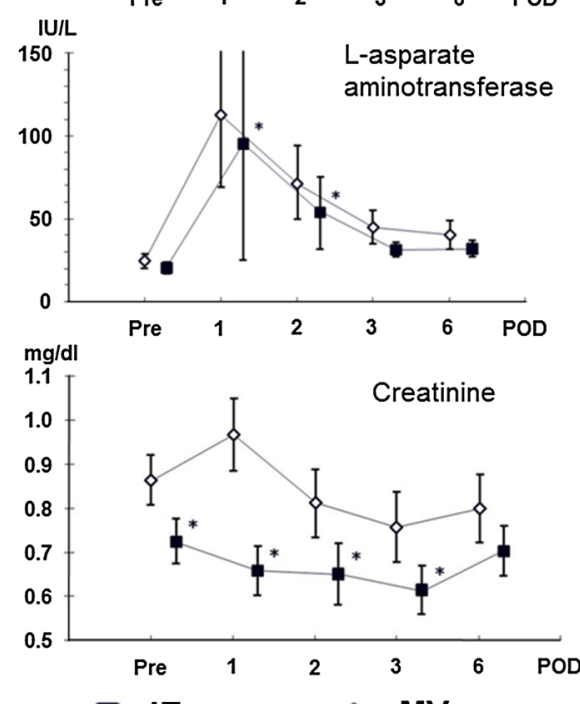

\section{Discussion}

This retrospective cohort study using a historical control with propensity score matching demonstrated that IE after transthoracic esophagectomy with radical 3-field lymphadenectomy for thoracic esophageal cancer facilitated postoperative recovery successfully with regard to early ambulation and length of ICU stay, with no significant differences in postoperative complication rates between groups.

Several reports have already demonstrated that IE after esophagectomy is feasible and safe in postoperative management [14-17]. However, the esophagectomies in these studies were mostly Ivor-Lewis and transhiatal esophagectomies. Only one study evaluated IE and MV after transthoracic esophagectomy with radical 3-field lymph node dissection. Toh et al., compared IE in the operating room and management of MV shortly after esophagectomy (average length of $\mathrm{MV}=9.9 \mathrm{~h}$ ) in patients after transthoracic esophagectomy with 3-field lymphadenectomy [18]. They demonstrated that the early postoperative clinical course was similar between the 2 groups. They also demonstrated that IE tended to reduce the incidence of pulmonary 
Table 4 Postoperative complications and in-hospital mortality

\begin{tabular}{|c|c|c|c|c|c|c|c|}
\hline & \multicolumn{3}{|c|}{ MV group $(n=48)$} & \multicolumn{3}{|c|}{ IE group $(n=48)$} & \multirow{2}{*}{$\frac{p}{0.404}$} \\
\hline All postoperative complications & $31(65 \%)$ & & & $27(56 \%$ & & & \\
\hline The Clavien-Dindo classification & I, II & III & IV & I, II & III & IV & \\
\hline Pneumonia & $8(17 \%)$ & $4(8 \%)$ & 0 & $2(4 \%)$ & $4(8 \%)$ & 0 & 0.229 \\
\hline Respiratory insufficiency & 0 & 0 & $4(8 \%)$ & 0 & 0 & $3(6 \%)$ & 0.694 \\
\hline Anastomotic leakage & 0 & $6(13 \%)$ & 0 & 0 & $4(8 \%)$ & 0 & 0.503 \\
\hline Recurrent nerve palsy & $4(8 \%)$ & $4(8 \%)$ & $1(2 \%)$ & $4(8 \%)$ & 0 & & 0.172 \\
\hline Arrhythmia & $3(6 \%)$ & 0 & & $2(4 \%)$ & 0 & & 0.645 \\
\hline Surgical site infections & 0 & $2(4 \%)$ & 0 & 0 & $1(2 \%)$ & 0 & 0.554 \\
\hline In-hospital mortality & $1(2 \%)$ & & & 0 & & & 1.000 \\
\hline
\end{tabular}

With overlaps

$M V$ overnight mechanical ventilation, $I E$ immediate extubation complications compared with MV. Our results are generally consistent with those of previous reports, and the rates of postoperative complications did not differ significantly between groups. Moreover, both the rate of re-intubation and the demand for bronchial toilet by fiber bronchoscopy were not significantly increased between groups. Therefore, IE may be feasible and safe, even after transthoracic esophagectomy with radical 3-field lymphadenectomy.

The present study demonstrated that early postoperative clinical outcomes are improved with IE. The decrease in postoperative inotrope use in the ICU was thought to be attributed to the lack of sedative use in the IE group, which may improve the patient's hemodynamic stability, thereby allowing early postoperative ambulation. Obviously, adequate postoperative analgesia was also essential to achieve early ambulation.

In this study, we changed only the timing of extubation, without any modification of other components of perioperative management, including routine follow-up duration in the ICU. Therefore, these factors may contribute to prolonging the median ICU stay for 3 days after surgery in the IE group. However, the rate of postoperative complications classified as Clavien-Dindo grade III or more was predisposed to be lower in the IE group compared to that in the MV group (Table 4). Therefore, the duration of ICU stay may be statistically shorter in the IE group compared to that in the MV group ( $p=0.01$ ), although the median ICU stay was equal in both groups.

In our study, we focused postoperative immunity and recovery from surgical stress. Smith and colleagues demonstrated that analgesic and sedative agents alter cellular function and other mediators of the immune system [21]. Galley and colleagues showed that anesthetics and sedatives modulate transport or secretion of IL-8, and suppression of IL- 8 by sedatives may predispose postoperative and intensive care patients to infection [22]. IE avoids the necessity of postoperative continuous infusion of sedative. Therefore, we hypothesized that IE would facilitate to early recovery from postoperative immunosuppressive conditions. However, changes in the peripheral white blood cell counts and lymphocyte counts were not significantly different between groups, On the other hand, the CRP level was significantly lower in the IE group than the MV group on postoperative day 1 . It was possibility that IE facilitate to early recovery from surgical stress.

Our results showed that the intraoperative in-out balance was significantly lower in the IE group than the MV group $(p=0.022)$. This finding suggests that anesthetists must pay attention to the intraoperative fluid balance to achieve IE safely after esophagectomy. Therefore, it remains possible that intraoperative fluid restriction may contribute to the clinical outcomes in the IE group. However, there is little evidence of the clinical usefulness of intraoperative fluid restriction in patients undergoing esophagectomy [23-25]. Goal-directed fluid therapy reduces postoperative pulmonary complications in major upper abdominal and vascular surgery [26]. However, few studies have assessed the role of goal-directed fluid therapy in esophagectomy. Further studies are needed to determine the optimal management of intraoperative fluid therapy in patients undergoing esophagectomy.

This study has several limitations. First, it was a retrospective study from only a single center. Second, it had a limited sample size and retrospective study with an inherent risk of bias. To some extent, hidden bias is inevitable, because the propensity score analysis controls only for observed and measured covariates. Therefore, prospective randomized controlled trials carried out in multiple centers are needed to validate these findings. Third, as patients in the IE group and the MV group underwent surgery during completely different time periods, the findings may have been influenced despite the application of case-matching.

In spite of these ambiguities, IE facilitated postoperative recovery successfully with regard to early ambulation and length of ICU stay. IE had no significant difference in postoperative complication rates. In the future, it would be 
interesting to carry out a cost analysis to examine if early extubation and earlier postoperative recovery also resulted in reduced costs after esophagectomy. We believe that IE is safe and feasible, even after transthoracic esophagectomy with radical 3-field lymphadenectomy for thoracic esophageal cancers, and should be a standard strategy for early postoperative respiratory management after radical esophagectomy.

Acknowledgements This study has no related financial support.

\section{Compliance with ethical standards}

Ethical statement The present study was approved by the review board of the Aichi Cancer Center Hospital. This study was also conducted in accordance with the guidelines of the Declaration of Helsinki (1964) and later versions.

Conflict of interest All authors declare that they have no conflict of interest.

\section{References}

1. Merritt RE, Whyte RI, D'Arcy NT, et al. Morbidity and mortality after esophagectomy following neoadjuvant chemoradiation. Ann Thorac Surg. 2011;92:2034-40.

2. Alldinger I, Sisic L, Hochreiter M, et al. Outcome, complications, and mortality of an intrathoracic anastomosis in esophageal cancer in patients without a preoperative selection with a risk score. Langenbecks Arch Surg. 2015;400:9-18.

3. O'Sullivan KE, Hurley ET, Hurley JP. Understanding complete pathologic response in oesophageal cancer: implications for management and survival. Gastroenterol Res Pract. 2015. https://doi. org/10.1155/2015/518281.

4. Avendano CE, Flume PA, Silvestri GA, et al. Pulmonary complications after esophagectomy. Ann Thorac Surg. 2002;73:922-6.

5. Atkins BZ, D'Amico TA. Respiratory complications after esophagectomy. Thorac Surg Clin. 2006;16:35-48.

6. van der Sluis PC, Verhage RJ, van der Horst S, et al. A new clinical scoring system to define pneumonia following esophagectomy for cancer. Dig Surg. 2014;31:108-16.

7. Li J, Shen Y, Tan L, et al. Is minimally invasive esophagectomy beneficial to elderly patients with esophageal cancer? Surg Endosc. 2015;29:925-30.

8. Weijs TJ, Ruurda JP, Nieuwenhuijzen GA, et al. Strategies to reduce pulmonary complications after esophagectomy. World J Gastroenterol. 2013;19:6509-14.

9. van Adrichem EJ, Meulenbroek RL, Plukker JT, et al. Comparison of two preoperative inspiratory muscle training programs to prevent pulmonary complications in patients undergoing esophagectomy: a randomized controlled pilot study. Ann Surg Oncol. 2014;21:2353-60.
10. Yoshida N, Baba Y, Hiyoshi Y, et al. Duration of smoking cessation and postoperative morbidity after esophagectomy for esophageal cancer: how long should patients stop smoking before surgery? World J Surg. 2016;40:142-7.

11. Patti MG, Wiener-Kronish JP, Way LW, et al. Impact of transhiatal esophagectomy on cardiac and respiratory function. Am J Surg. 1991;162:563-6.

12. Forshaw MJ, Khan AZ, Davies AR, et al. Postoperative ventilation in the recovery area. Ann R Coll Surg Engl. 2007;89:449.

13. Caldwell MT, Murphy PG, Page R, et al. Timing of extubation after oesophagectomy. Br J Surg. 1993;80:1537-9.

14. Yap FH, Lau JY, Joynt GM, et al. Early extubation after transthoracic oesophagectomy. Hong Kong Med J. 2003;9:98-102.

15. Chandrashekar MV, Irving M, Wayman J, et al. Immediate extubation and epidural analgesia allow safe management in a highdependency unit after two-stage oesophagectomy. Results of 8 years of experience in a specialized upper gastrointestinal unit in a district general hospital. Br J Anaesth. 2003;90:474-9.

16. Robertson SA, Skipworth RJ, Clarke DL, et al. Ventilatory and intensive care requirements following oesophageal resection. Ann R Coll Surg Engl. 2006;88:354-7.

17. Lanuti M, de Delva PE, Maher A, et al. Feasibility and outcomes of an early extubation policy after esophagectomy. Ann Thorac Surg. 2006;82:2037-41.

18. Toh Y, Oki E, Minami K, et al. Evaluation of the feasibility and safety of immediate extubation after esophagectomy with extended radical three-field lymph node dissection for thoracic esophageal cancers. Esophagus. 2009;6:167-72.

19. Abe T, Hatooka S, Niwa Y, et al. Invention of the enteral feeding catheter insertion method at esophagectomy with gastric tube reconstruction. Syujutsu (Operation). 2009;65:589-92 (in Japanese).

20. Sobin LH, Gospodarowicz MK, Wittekind CH. TNM classification of malignant tumours. 7th ed. Oxford: Wiley-Blackwell; 2009.

21. Smith MA, Hibino M, Falcione BA, et al. Immunosuppressive aspects of analgesics and sedatives used in mechanically ventilated patients: an underappreciated risk factor for the development of ventilator-associated pneumonia in critically ill patients. Ann Pharmacother. 2014;48:77-85.

22. Galley HF, Dubbels AM, Webster NR. The effect of midazolam and propofol on interleukin- 8 from human polymorphonuclear leukocytes. Anesth Analg. 1998;86:1289-93.

23. Kita T, Mammoto T, Kishi Y. Fluid management and postoperative respiratory disturbances in patients with transthoracic esophagectomy for carcinoma. J Clin Anesth. 2002;14:252-6.

24. Brandstrup B. Fluid therapy for the surgical patient. Best Pract Res Clin Anaesthesiol. 2006;20:265-83.

25. Buise M, Van Bommel J, Mehra M, et al. Pulmonary morbidity following esophagectomy is decreased after introduction of a multimodal anesthetic regimen. Acta Anaesthesiol Belg. 2008;59:257-61.

26. Corcoran T, Rhodes JE, Clarke S, et al. Perioperative fluid management strategies in major surgery: a stratified meta-analysis. Anesth Analg. 2012;114:640-51. 